Projets

de paysage

\section{Projets de paysage}

Revue scientifique sur la conception et l'aménagement de l'espace

$17 \mid 2017$

Paysage(s) et agriculture(s)

\title{
Les territoires agriurbains en Île-de-France : entre paysage ordinaire, paysage agricole et paysage alimentaire?
}

Agri-Urban Areas in the Île-de-France Region: Between Ordinary, Agricultural and Food-Producing Landscapes?

\section{Monique Toublanc et Monique Poulot}

\section{(2) OpenEdition}

\section{Journals}

Édition électronique

URL : http://journals.openedition.org/paysage/4782

DOI : $10.4000 /$ paysage.4782

ISSN : 1969-6124

Éditeur :

École nationale supérieure du paysage de Versailles-Marseille, Institut national des sciences appliquées Centre Val de Loire - École de la nature et du paysage, École nationale supérieure d'architecture et de paysage de Bordeaux, École nationale supérieure d'architecture et de paysage de Lille, Agrocampus Angers

Référence électronique

Monique Toublanc et Monique Poulot, «Les territoires agriurbains en Île-de-France : entre paysage ordinaire, paysage agricole et paysage alimentaire ? », Projets de paysage [En ligne], 17 | 2017, mis en ligne le, consulté le 03 avril 2020. URL : http://journals.openedition.org/paysage/4782 ; DOI : https:// doi.org/10.4000/paysage.4782

Ce document a été généré automatiquement le 3 avril 2020.

Projets de paysage 


\title{
Les territoires agriurbains en Île-de- France : entre paysage ordinaire, paysage agricole et paysage alimentaire?
}

\author{
Agri-Urban Areas in the Île-de-France Region: Between Ordinary, Agricultural \\ and Food-Producing Landscapes?
}

Monique Toublanc et Monique Poulot

1 Les programmes agriurbains, derniers nés (1999) des territoires de projet en région Îlede-France (RIF), consacrent la prise en compte des espaces agricoles et de leurs paysages dans la construction de l'urbain. Dispositifs éprouvés de manière informelle dès les années $1970^{1}$, ils reçoivent une reconnaissance officielle en juillet 2001 quand le Comité interministériel d'aménagement et de développement du territoire décide d'un accompagnement financier pour sept d'entre eux, avant d'être institutionnalisés dans la loi relative au développement des territoires ruraux (février 2005). Devenus partie intégrante des systèmes métropolitains, les espaces « ouverts » agricoles ayant acquis une valeur pour les habitants sont désormais pensés comme nécessaires «à leur bonne santé » (Poulot, 2013). Ils s'imposent au moins dans les débats et les discours, car inscrits dans le référentiel de la ville durable qui fonde aujourd'hui la vision de ceux qui la construisent. Ourlant le front de l'avancée urbaine en ce début de $\mathrm{xxI}^{\mathrm{e}}$ siècle, ces espaces épousent le contour de territoires reconnus pour certains «territoires agriurbains» (TAU) et soutenus financièrement depuis 2007 par la RIF : des acteurs (agriculteurs, collectivités territoriales, habitants et associations) s'emploient à y faire converger ville et agriculture dans un même projet de territoire.

2 Les TAU ne correspondent nullement à des délimitations institutionnelles (par exemple communautés de communes et/ou d'agglomération) mais réunissent dans une même enveloppe spatiale des communes offrant les trois types d'espaces franciliens définis par l'Institut d'aménagement et d'urbanisme de la région Île-de-France (IAU) selon une approche morphologique: de l'urbain dense, des zones sous influence de 
l'agglomération centrale offrant de 20 à $55 \%$ d'espaces bâtis, enfin des espaces à dominante rurale occupés à $80 \%$ par des cultures et/ou des forêts (Direction régionale interdépartementale de l'alimentation, de l'agriculture et de la forêt /DRIAAF-IAU, 2004). Ils peuvent ainsi être appréhendés comme une forme de territorialisation où s'affirmerait une volonté de (re)qualification paysagère s'appuyant sur des ressources territoriales propres dans des espaces ordinaires, mêlant bâti et non-bâti sous les différentes poussées de la périurbanisation (Berger, 2004). Cette évolution participe d'un processus général de reconnaissance des paysages dits ordinaires (vs paysages exceptionnels) à l'œuvre depuis trois décennies en France, corrélatif d'une transformation de la notion de paysage entendue aujourd'hui comme " une relation, une modalité particulière [sensible et symbolique] de la relation de la société à son environnement » (Berque, 1991). Désignés par l'expression "paysages sans qualités », par le qualificatif de "banals", ni remarqués - sauf pour les déprécier ${ }^{2}$ - ni remarquables (pas d'images reconnues et revendiquées mettant en avant leur caractère), les TAU sont le théâtre d'une dynamique prenant à rebours les représentations paysagères négatives communément diffusées à leur adresse. Parce qu'ils sont habités, donc perçus à travers des usages répétés tissant par la force de l'habitude des liens affectifs souvent forts entre des individus et leur milieu de vie, ils ont vocation, aux yeux des chercheurs et des professionnels de l'aménagement de l'espace, à être pris en charge comme " paysages de tous les jours ».

Partageant une même situation géographique métropolitaine et une histoire, si ce n'est commune tout au moins proche, liée au développement de la région parisienne (figure 1), ils sont aujourd'hui en réseau sous l'égide de la Bergerie nationale qui en sa qualité de centre de ressources sur le développement durable en est l'animateur. Ils affichent une volonté commune: affirmer leur spécificité vis-à-vis de l'extérieur et conduire les habitants et les acteurs économiques à se reconnaitre dans ces territoires et à s'y identifier (sentiment d'appartenance partagé). Pour ce faire, les protagonistes conduisent selon des modalités de pilotage collectif des programmes d'actions dits agriurbains participant à la fois du projet de territoire, du projet de société et du projet de paysage (Poulot, 2008). Alors que les TAU ont été longtemps centrés sur les deux registres, agricole et urbain, la question alimentaire y est aujourd'hui brandie de façon explicite et pour ainsi dire autonome ; elle est devenue un élément clef des discours des acteurs de la société civile et des politiques urbaines (Steel, 2016), déclinée à toutes les échelles spatiales, du national ou local (Lardon et Loudiyi, 2014). 
Figure 1. À l'ouest et au sud, le front urbain parisien est adossé aux territoires agriurbains

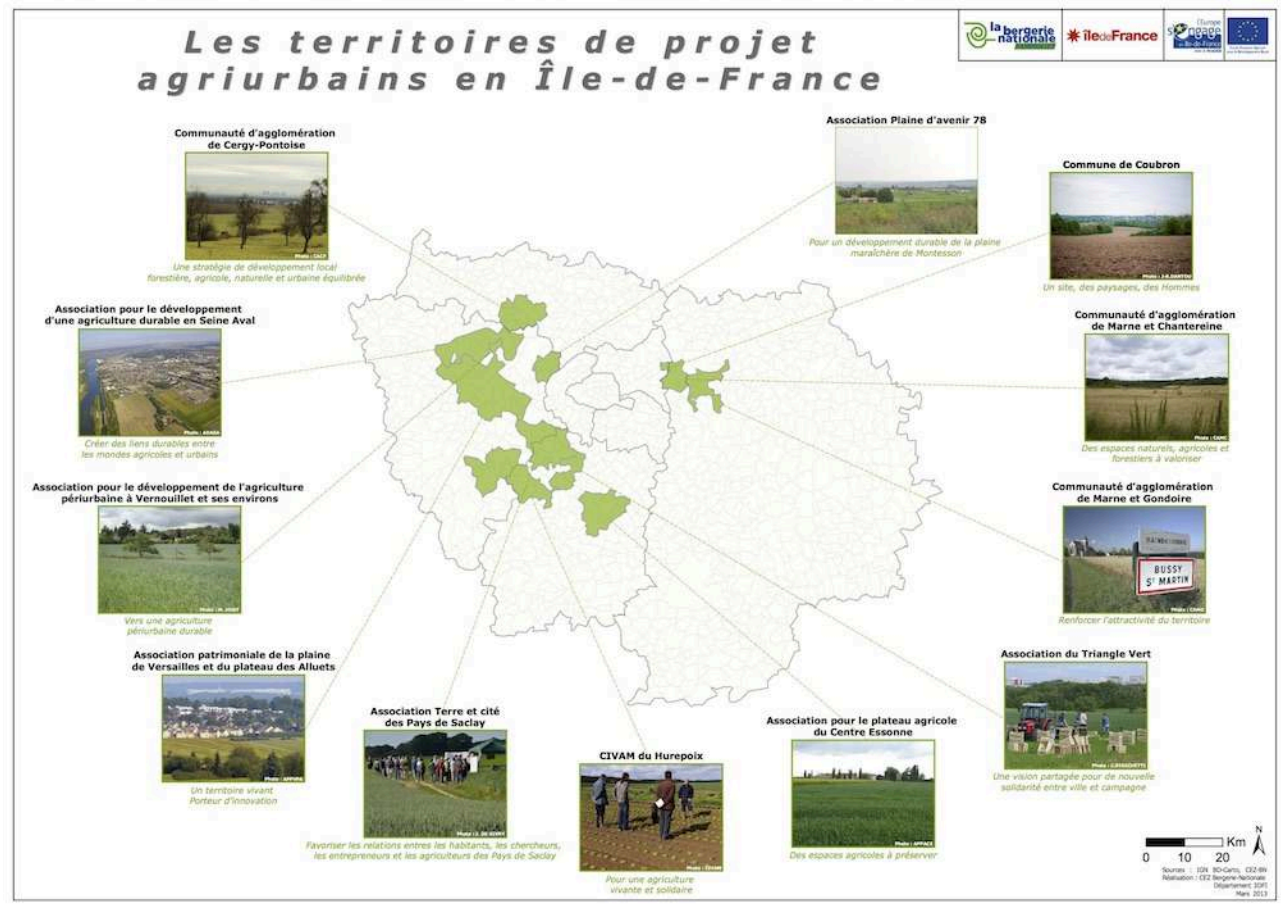

4 Nous posons l'hypothèse que le consensus que tentent aujourd'hui d'atteindre les acteurs sur les TAU porte (et est porté par) un projet de paysage, entre paysages urbain, agricole et, de plus en plus, alimentaire. La notion de projet de paysage, envisagé comme un dessin autant qu'un dessein (Donadieu et Mazas, 2002), permet de rassembler la quasi-totalité des actions menées (tableau 1).

Tableau 1. Des actions pour tisser ensemble agriculture et bâti et donner à voir les territoires

\begin{tabular}{|c|c|c|c|}
\hline \multicolumn{4}{|l|}{ Registres d'actions } \\
\hline $\begin{array}{lr}\text { Maintien } & \text { et } \\
\text { développement } & \text { des } \\
\text { activités agricoles } & \end{array}$ & $\begin{array}{lr}\text { Intervention } & \text { pour } \\
\text { l'amélioration } & \text { des } \\
\text { conditions } & \\
\text { d'exploitation } & \end{array}$ & $\begin{array}{l}\text { Appui aux projets de } \\
\text { développement des } \\
\text { exploitations agricoles }\end{array}$ & $\begin{array}{ll}\text { Promotion } & \text { des } \\
\text { exploitations et } & \text { des } \\
\text { produits agricoles } & \end{array}$ \\
\hline 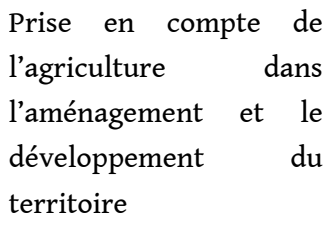 & $\begin{array}{l}\text { Mobilisation et } \\
\text { accompagnement des } \\
\text { acteurs locaux }\end{array}$ & $\begin{array}{l}\text { Élaboration et } \\
\text { animation de projets } \\
\text { agricoles de territoire } \\
\text { en concertation }\end{array}$ & $\begin{array}{l}\text { Prospection et } \\
\text { approfondissement des } \\
\text { connaissances }\end{array}$ \\
\hline 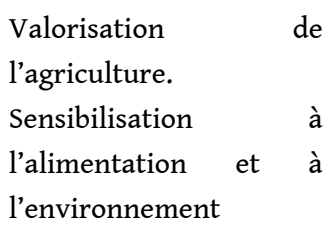 & $\begin{array}{l}\text { Organisation } \\
\text { d'événements } \\
\text { d'animations }\end{array}$ & $\begin{array}{l}\text { Création d'outils } \\
\text { pédagogiques et de } \\
\text { communication }\end{array}$ & $\begin{array}{l}\text { Accompagnement des } \\
\text { acteurs locaux }\end{array}$ \\
\hline
\end{tabular}

Source : Bergerie nationale, « Les programmes agriurbains d'Île-de-France. État des lieux, bilan et perspectives. Rapport d'étude », avril 2016, 112 p. 
Quelle(s) figure(s) territoriale(s) et paysagère(s) est (sont) promue (s) dans les TAU ? En quoi le débat sur l'alimentation les modifie-t-elle(s)? Notre réflexion s'appuie sur l'un des programmes de recherche « Pour et sur le développement régional (PSDR) » ̂̂le-deFrance (IDF) nommé «Archipels agriurbains, résistances et gouvernances » (Agrige ${ }^{3}$ ) mais elle s'ancre également sur d'autres réflexions issues de recherches ${ }^{4}$ menées auparavant par les auteures. L'article repose sur une analyse croisée des discours (littérature grise, sites Internet, enquête...) produits par les différentes parties prenantes des TAU (élus, agriculteurs, habitants, animateurs, élus, aménageurs...) et sur une étude des actions en cours ou programmées ${ }^{5}$ par les TAU, examinées sous l'angle du paysage.

6 Nous présenterons d'abord un panel d'actions ou de discours mis en œuvre par les acteurs locaux dans les TAU avant d'analyser, dans leur double dimension matérielle et idéelle, deux figures paysagères emblématiques. La seconde partie portera sur l'introduction à la fois en actes et en mots de la préoccupation alimentaire dans les TAU et son lien au paysage. La conclusion interrogera comment cette dimension alimentaire, nouvelle sur un pas de temps de $30 \mathrm{ans}^{6}$, amène à revisiter et à infléchir le projet agriurbain initial (« l'agriurbanisme » des années 1990 : Vidal et Fleury, 2007) et, ce faisant, participe à redéfinir, voire à réinventer l'activité agricole, le métier d'agriculteur et son identité professionnelle.

\section{Des figures paysagères emblématiques de l'agriurbanisme}

\section{L'agriurbanisme : quel projet paysager?}

$7 \quad$ Loin des seules pratiques de zonages décrétés selon un strict partage des activités, les TAU sont des laboratoires marqués par l'affichage d'un consensus le plus large possible, mais non exempt de conflits, rassemblant les différentes parties prenantes (habitants, agriculteurs, acteurs institutionnels, et associatifs) autour d'un projet de territoire agriurbain qui conjugue développement urbain et promotion de l'agriculture en tant que bien public commun : en somme, un paysage hybride où les espaces ouverts ont droit de cité et sont même censés devenir structurants. Les TAU sont portés par la signature de contrats à différentes échelles et le déploiement de programmes d'actions, estampillées agriurbaines, ayant pour finalités, une intégration de logiques longtemps considérées comme contradictoires - urbaine et agricole, environnementale et économique - et la recherche de nouvelles articulations entre monde agricole et société urbaine (Chalas, 2005). Cette interdépendance repose sur une gouvernance transversale, inventée pour l'occasion, avec la création de trois ou quatre collèges regroupant qui les élus, qui les agriculteurs, qui les associations et/ou les habitants afin que les décisions prises et les actions conduites le soient de façon concertée et coordonnée (Poulot, 2008 et 2014). Enfin un animateur, le plus souvent à temps partiel, accompagne la réalisation des programmes d'action et veille à ce que des liens se tissent entre les différentes catégories d'acteurs du territoire.

8 L'analyse des actions conduites par les TAU (tableau $n^{\circ} 1$ ) montre trois formes d'intervention: "maintien et développement des activités agricoles", "prise en compte de l'agriculture dans l'aménagement et le développement du territoire", «valorisation de l'agriculture. Sensibilisation à l'alimentation et à l'environnement » 
(Bergerie nationale, 2016). Les premières, qui ciblent agriculteurs et exploitations agricoles, visent à préserver et à développer l'activité agricole, notamment en agissant sur le foncier : elles sont le fait de la puissance publique (Région Île-de-France et autres collectivités territoriales) mais aussi d'initiatives citoyennes (Poulot, 2014). Les secondes engagent les collectivités dans la construction de projets agricoles territorialisés qui soient force de proposition face aux processus et aux politiques d'urbanisation: elles appellent surtout des actions économiques en faveur d'une agriculture en circuits courts. Les dernières relèvent du champ de la médiation et de l'éducation et s'adressent aux habitants non-agriculteurs et à tous les acteurs du territoire, collectivités comprises : de nature didactique, elles embrassent le registre du symbolique et reposent sur des valeurs.

9 Les animateurs-médiateurs et acteurs enquêtés, arguant notamment du lien à la capitale et de son emprise sur les TAU, estiment que les actions qu'ils conduisent sont différentes de celles menées sur des territoires ruraux ordinaires en ce qu'elles tiennent compte de la proximité urbaine dans un jeu complexe, entre contraintes et opportunités. Peut-on dire qu'elles façonnent des paysages " originaux »? Autrement dit, fabriquent-elles des paysages singuliers en transformant la matérialité du paysage (sa réalité territoriale), et en projetant sur celle-ci des images spécifiques susceptibles de construire une (ou des) représentation(s) paysagère(s) ? La réponse est complexe mais il paraît possible de repérer des modes d'intervention (ou des intentions) spécifiques et récurrents: l'aménagement des situations de contact entre l'espace urbain bâti et l'espace agricole qui l'environne (exemples: plaine de Versailles, commune de Villepreux, frange nord), la création de sentiers d'interprétation agricole (exemples : plateau de Saclay, Gâtinais français, plateau Briard) ou encore la mise en œuvre de nombreuses actions de communication... Ces projets visent à créer des liens visuels, fonctionnels et sociaux, entre des espaces juxtaposés (terres agricoles et ville) qui le plus souvent s'ignorent et cherchent à apprendre aux deux mondes agricole et urbain à se connaître et à échanger.

Pour penser et développer leurs programmes d'actions, les acteurs choisissent dans la matrice paysagère et territoriale des TAU des appuis, des «prises » (Berque, 2000). Ces points d'accroche varieront selon les caractéristiques agricoles des TAU, plus ou moins accordées avec les spécificités topographiques et pédologiques: type de culture, répartition des différentes soles et organisation du parcellaire, localisation des sièges d'exploitation, rapport spatial aux secteurs urbanisés et aux territoires ruraux, situations enclavées ou ouvertes sur d'autres espaces agricoles, ancienneté de la dynamique agriurbaine, etc. Une même action n'aura donc pas le même effet d'un TAU à un autre. Les exemples de la plaine de Montesson (Yvelines) et de Coubron (SeineSaint-Denis) - TAU entré en sommeil depuis deux ans - témoignent de cette variabilité : ainsi la plaine de Montesson, nichée dans un méandre de la Seine, accueille des cultures maraîchères de plein champ dans le cadre de grandes exploitations ayant œuvré pour un paysage ouvert avec un report des sièges d'exploitation sur les bordures, quand le secteur de Coubron embrasse les coteaux d'Aulnoye avec, sur les prairies calcicoles, un élevage bovin et caprin et, sur les pentes, des vergers et des vignes. Dans le premier cas, la quasi-totalité des arbres ayant été supprimée pour gagner de la terre arable, les acteurs travaillent surtout sur une intégration de la plaine de Montesson dans le grand paysage de l'ouest francilien, jouant d'une position centrale entre les terrasses de Saint-Germain-en-Laye et La Défense. En revanche, à Coubron, situé à deux pas de fortes densités urbaines et d'un bâti relativement dégradé en Seine-Saint-Denis, 
l'enfrichement et le reboisement ont gagné tant le contexte était peu favorable à une agriculture productiviste, et les actions paysagères ont pour point d'ancrage les vues sur la Seine et les formes bocagères résiduelles.

11 Plutôt que de considérer «la politique agriurbaine » des TAU et ses effets paysagers, nous avons privilégié deux objets, paysagistes et urbanistiques: le sentier d'interprétation agricole et la lisière urbaine. Ils nous semblent en effet, si ce n'est spécifiques, du moins emblématiques des TAU puisque projetés de façon récurrente dans les programmes d'actions. On en interrogera notamment la traduction paysagère au sens plein du terme.

\section{À la découverte du paysage agriurbain : le sentier d'interprétation agricole $^{7}$}

12 Ce dispositif s'impose pour les TAU comme un outil de référence pour répondre à la problématique du lien entre l'urbain et l'agriculture. Pourtant, seuls trois sentiers, inaugurés entre 2005 et 2006, ont été réalisés en Île-de-France sous la houlette de la Bergerie nationale (Guiomar, 2010). Les deux premiers sont inclus dans un TAU, soit existant (plateau de Saclay) soit en réactivation (le plateau Briard), quand le dernier est dans un Parc naturel régional (Gâtinais français) : les territoires d'implantation ont été choisis en raison de leur diversité agricole, d'un potentiel important de fréquentation, de la présence de pôles d'animation (une ferme ouverte au public, un musée...) perçus comme attractifs et figurant des accroches en puissance pour les sentiers. Financées pour l'essentiel par un programme européen (Sustainable Open Spaces II, 2000-2004), inspirées d'actions conduites en Angleterre dans les années 1990 et établies sur une interdépendance ville-campagne (Salomon Cavin, 2006), ces formes de cheminement au milieu de terres agricoles, devenues symboliquement publiques, se veulent des outils de médiation entre la ville et l'agriculture.

13 La création de tels sentiers se fonde sur le postulat d'une méconnaissance du monde agricole par ceux qui n'en sont pas les acteurs, autrement dit, les «citadins». Ces derniers, habitant ou non dans ces territoires, en auraient une vision soit passéiste, soit négative (Bataille, 2002) et ne posséderaient pas les clés de lecture leur permettant de mesurer la valeur des espaces façonnés par l'activité agricole. Ponctué de panneaux didactiques, le cheminement proposé est pensé comme une expérience interactive destinée à montrer que "l'agriculture est garante, en bordure de ville, d'un espace écologiquement et culturellement sain, riche et porteur d'identité ; qu'elle contribue à la présence de repères sociotemporels, aux loisirs, au recyclage, à la protection d'un environnement spécifique en plus d'une production de qualité ; qu'elle est solidaire de l'avenir des territoires périurbains, espaces de transition et en transition à la recherche de territorialités » (Guiomar, 2010). La finalité du sentier est multiple : aider le public, habitant ou de passage, à prendre conscience de l'existence d'un risque, celui de voir disparaître ces espaces sous l'effet de l'urbanisation ; l'alerter sur l'existence de motifs paysagers, perçus comme menacés à plus ou moins long terme; lui apprendre à apprécier, à ressentir un lieu et ce que celui-ci doit à l'agriculture pour ensuite chercher à la protéger.

Les trois sentiers franciliens courent sur 4 à 8 kilomètres chacun et veulent promouvoir l'image d'une agriculture de proximité, présentée comme garante d'une bonne gestion de l'environnement et du patrimoine (figure 2). Les panneaux, une petite vingtaine, 
conçus et rédigés en concertation entre les différents acteurs (agriculteurs, associations, collectivités locales), interrogent le marcheur sur ce qu'il voit et apportent des éléments de réponse en surlignant les éléments révélateurs des interactions entre agriculture et ville. Les thèmes abordés traitent des composantes du paysage et de l'environnement (faune, flore, biodiversité, haies, mares, systèmes de drainage et d'irrigation, architecture, aménagement du territoire...), des logiques agricoles - y compris les systèmes d'aides publiques -, et de certaines caractéristiques ayant trait à l'alimentation (saisonnalité des produits agricoles, variétés proposées...). Dans tous les cas, il s'agit d'amener le promeneur à une identification de l'agriculteur : comme producteur ici même d'aliments de qualité ${ }^{8}$ et de paysage dans une relation permanente à la nature ; comme agent du développement économique local (emplois directs et indirects, vente de produits agricoles...); comme partenaire de la préservation de l'environnement (plantation de haies, curage des fossés, drainage...) ; et comme acteur de l'entretien des dispositifs permettant la fréquentation touristique (chemins, points de vue, patrimoine rural). Ces sentiers s'appuient sur quelques lieux plus symboliques que d'autres pour affirmer la dimension historique et identitaire de l'agriculture : le Conservatoire national des plantes à parfum, médicinales, aromatiques et industrielles, installé à Milly-la-Forêt, un des trois sites remarquables du goût de l'île-de-France ${ }^{9}$; l'ancienne gare aux roses de Mandres-les-Roses transformée en ferme d'animation gérée par un Centre d'Aide par le Travail ; le système des étangs du plateau de Saclay destiné à l'alimentation du parc de Versailles. Enfin, ils proposent la visite d'une ferme d'accueil ou de cueillette (la ferme de Viltain par exemple) et font plus ou moins le lien avec le versant moderne et industriel de l'agriculture (comme les établissements Darégal-Darbonne ${ }^{10}$ à Milly-la-Forêt).

Quelques années plus tard, le bilan est plutôt mitigé. Si les panneaux traitent de l'agriculture, jouent-ils le rôle d'un vecteur rapprochant les agriculteurs et les promeneurs? Partant du constat qu'il est rare de rencontrer des agriculteurs et donc de pouvoir amorcer avec eux un échange verbal, les auteurs ont pensé la signalétique à la manière d'un dialogue agriculteurs/promeneurs ; mais si riche soit l'information sur les panneaux, celle-ci n'est guère orientée vers le paysage agricole traversé ou l'exploitation agricole qui façonne celui-ci, ici et maintenant, mais vers l'agriculture en général, à tel point que le sentier peut paraitre déterritorialisé. De fait, « l'information seule n'est pas de l'interprétation » (Tilden et al., 2008). Certains panneaux n'instaurent pas une situation d'échange ou de proximité avec les lieux alentour et ils pourraient être implantés n'importe où ailleurs. D'autres sont porteurs d'un message historique et véhiculent une vision patrimoniale de l'agriculture très éloignée de la réalité présente : outre qu'ils s'intéressent principalement au bâti ancien sans développer un récit historique élargi à l'agriculture dans son ensemble, ils n'articulent pas les trois temps passé/présent/avenir sur des territoires, qui présentent pourtant les traces des systèmes anciens à la manière d'un palimpseste. Enfin, le thème de la ville et du développement urbain est finalement très peu présent. On peut s'interroger sur l'efficience paysagère de sentiers qui ne " conversent » pas vraiment avec le territoire dans lequel ils s'inscrivent: ainsi conçus et équipés, les cheminements proposés offrent-ils aux promeneurs la possibilité de nouer une relation sensorielle, émotionnelle, imaginaire, avec l'environnement agricole qu'ils sillonnent et qu'ils traversent, de vivre une expérience paysagère au sens phénoménologique du terme, de se forger une opinion et des convictions à l'endroit du territoire arpenté ${ }^{11}$ ? 
Figure 2a. Les sentiers d'interprétation agricole à l'épreuve du temps

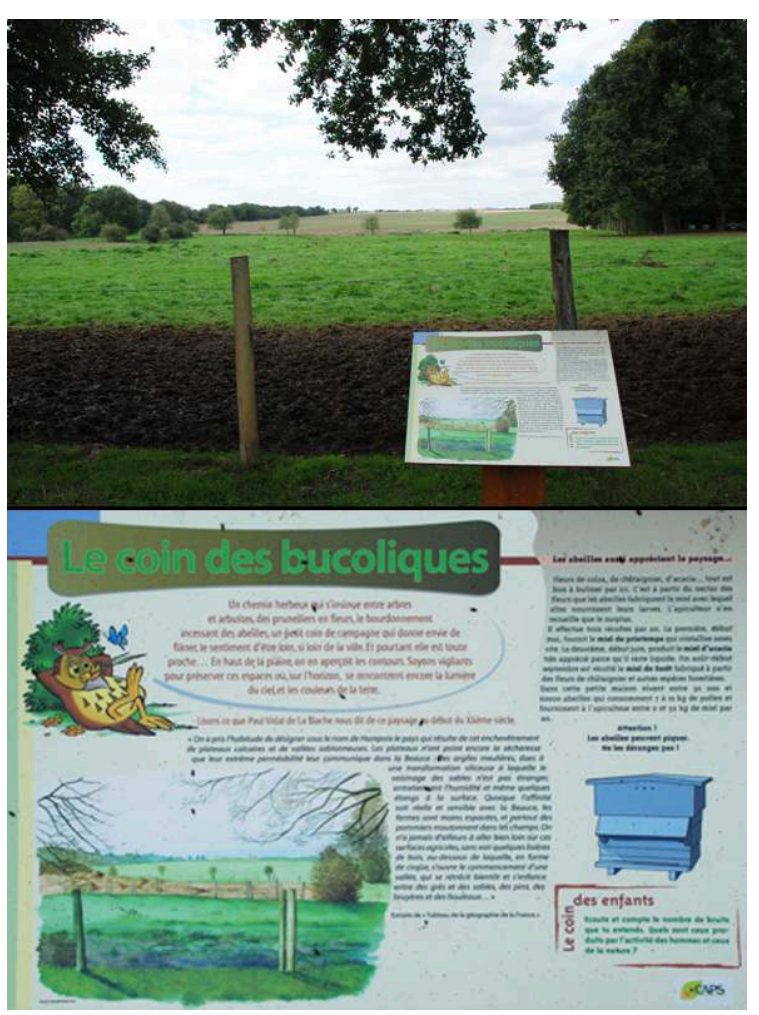

La «belle campagne » : une vision agreste de « l'espace ouvert » modelé par l'agriculture. Le sentier d'interprétation agricole du plateau de Saclay.

Les quelques panneaux choisis montrent les thématiques illustrées, les difficultés à révéler les lieux (style, décalages temporels et spatiaux) et l'absence d'entretien.

Source : Anne Genest, 2015 
Figure $2 \mathrm{~b}$. Les sentiers d'interprétation agricole à l'épreuve du temps

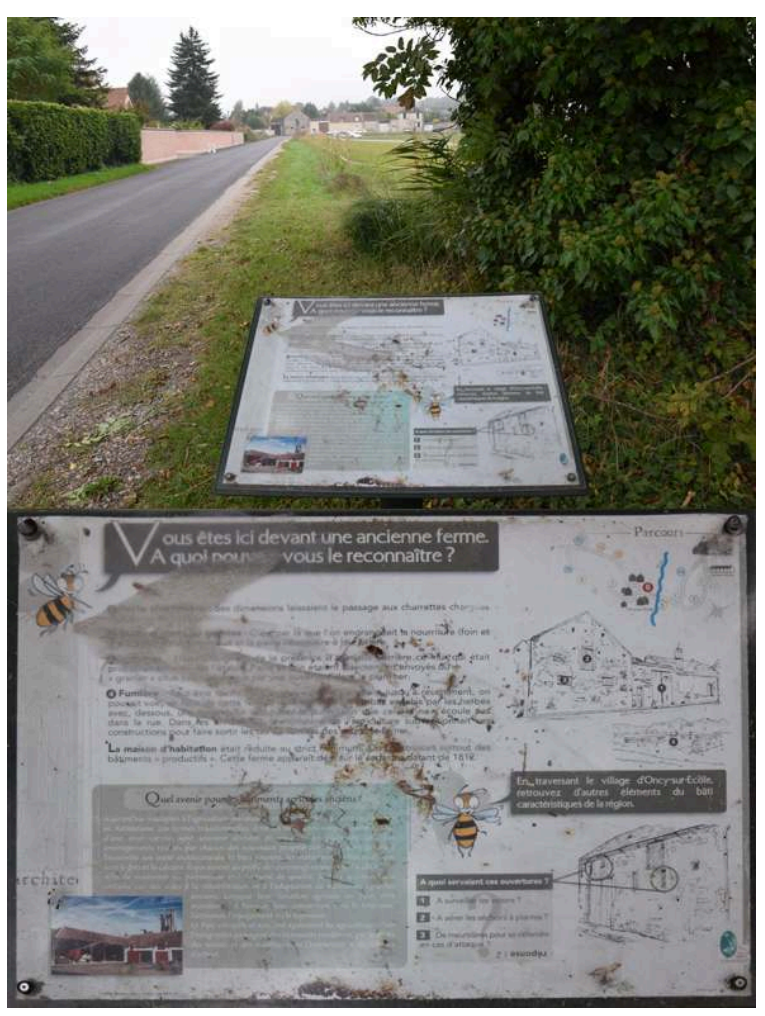

Un regard patrimonial sur le bâti agricole. Le sentier d'interprétation agricole de Milly-la-Forêt (Gâtinais français).

Source : Anne Genest, 2015. 
Figure 2c. Les sentiers d'interprétation agricole à l'épreuve du temps

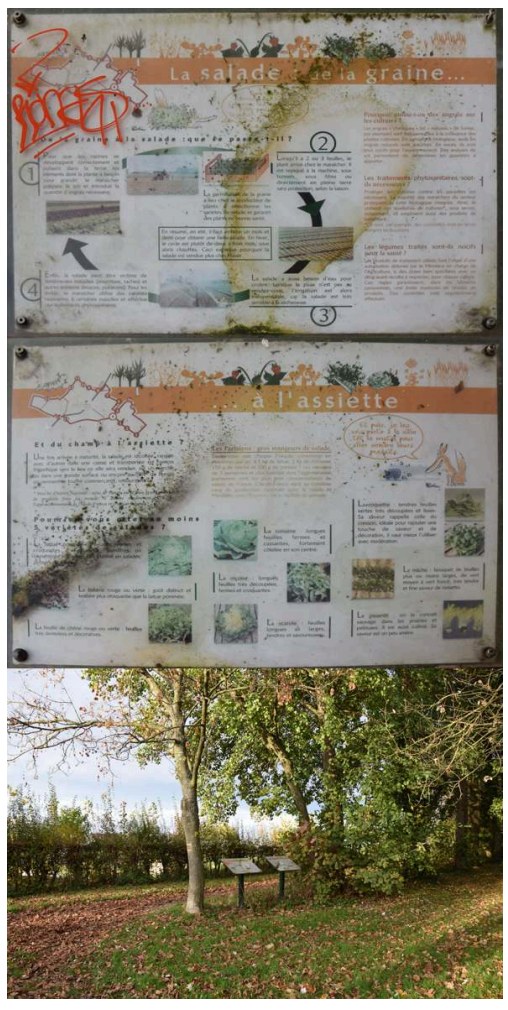

Le paysage nourricier du domaine de Saint-Leu. Derrière la haie et les panneaux, les parcelles maraîchères du domaine de Saint-Leu. Le sentier d'interprétation agricole de Périgny-sur-Yerres (plateau Briard).

Source : Anne Genest, 2015 
Figure $2 \mathrm{~d}$. Les sentiers d'interprétation agricole à l'épreuve du temps

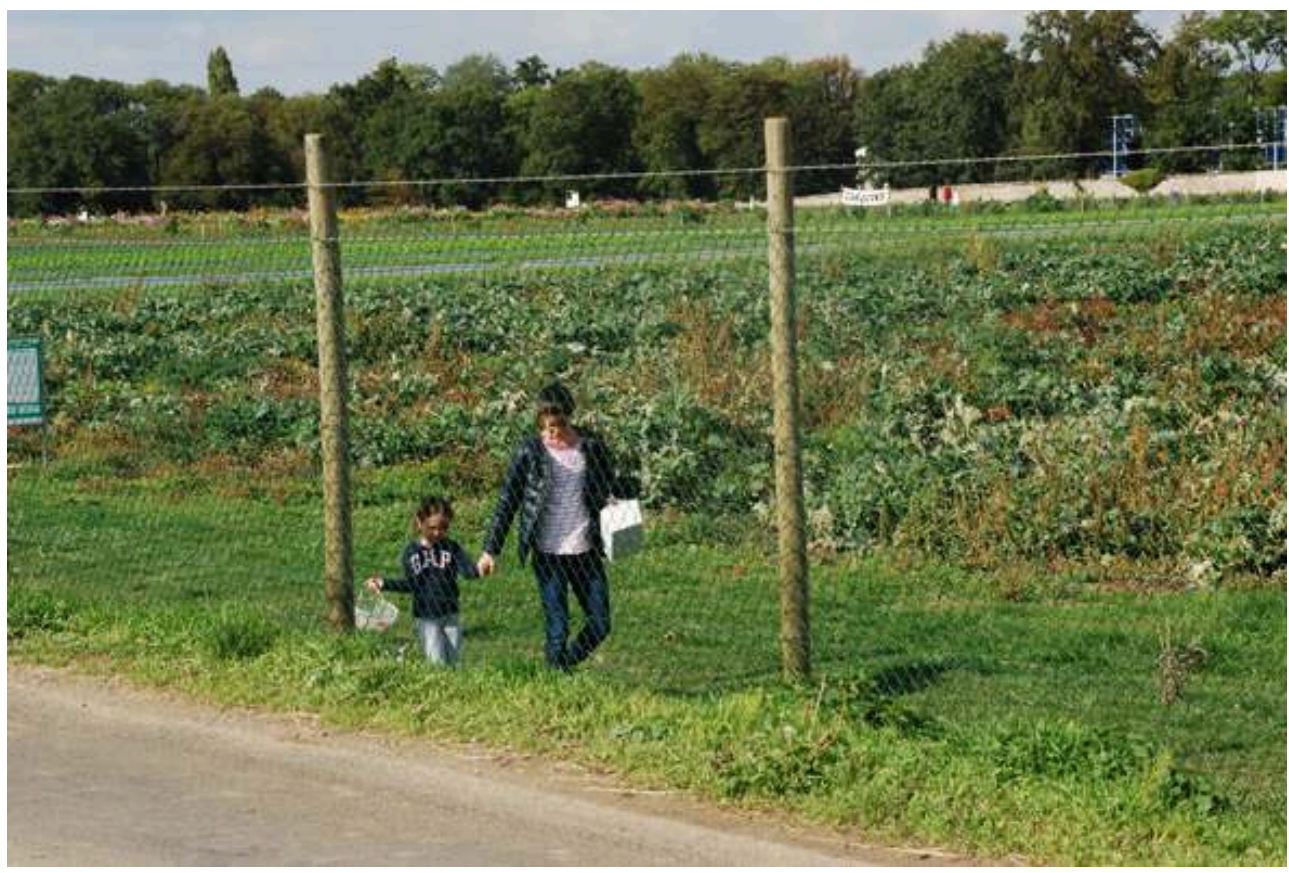

Récolte à la ferme de Viltain : des citadins à la rencontre du maraîchage. Le sentier d'interprétation agricole du plateau de Saclay (Yvelines).

Source : Anne Genest, 2015

De surcroît, créés il y a une dizaine d'années, les dispositifs ne sont pas entretenus et se dégradent: vues fermées par la végétation, panneaux inaccessibles enfouis dans les herbes hautes et devenus illisibles pour certains (supports abîmés par le temps, la malveillance ou le vandalisme). De telles évolutions témoignent d'une usure matérielle mais posent surtout la question de la fréquentation des sentiers: selon toute apparence, si les lieux (fermes notamment) qu'ils relient connaissent des visites, le cheminement ne paraît pas très important et les promeneurs peu nombreux ; beaucoup utilisés au départ par les écoles, ils semblent l'être moins aujourd'hui. Enfin, ne peut-on pas lire ici l'écho de l'obsolescence, peut-être inévitable, de ce type de dispositif dès lors que son objet, le territoire (et l'agriculture), est en mouvement et se transforme sans cesse : la question de trouver des outils susceptibles d'enregistrer les transformations des lieux est d'ailleurs à l'ordre du jour dans les projets de nouveaux sentiers (ainsi en plaine de Montesson).

\section{Un marqueur paysager de l'agriurbanisme : la lisière urbaine}

17 La lisière urbaine au sens où l'entendent des professionnels de l'aménagement et de la planification du territoire est un concept faisant advenir une autre figure pour ainsi dire générique du projet de paysage appliquée aux TAU. Cette notion a été inventée par les concepteurs, notamment les paysagistes, pour penser les situations de contact entre l'espace urbain bâti et l'espace qui le borde, «naturel » ou agricole (Beringuier et al., 2015; Bonin, 2013) en organisant (spatialement) des interfaces, des transitions (Toublanc et al., 2016) : si l'expression est relativement récente, la préoccupation des limites spatiales est ancienne, récurrente et fondatrice de la pratique paysagiste en France. Sans relâche, les professionnels du paysage (Bertrand Folléa, Michel Desvigne 
pour ne citer qu'eux) affirment la nécessité de "recoudre " un territoire qu'ils perçoivent comme morcelé, de transgresser les «frontières" qui le traversent, de restaurer des relations harmonieuses entre des espaces qu'ils considèrent comme cloisonnés.

Cette rhétorique trouve un terrain topique dans les TAU ici étudiés. Découpés, entaillés, zébrés par de nombreuses infrastructures de transport (énergétiques, routiers, ferroviaires...), les TAU sont fracturés de toute part et offrent des ambiances très contrastées entre des systèmes spatiaux qui s'interrompent brusquement: centrebourgs à l'architecture rurale, grandes cultures ou cultures spéciales, réseaux de communication (autoroutes, TGV, routes, chemins communaux...) y alternent, produisant un paysage patchwork dont la notion de limite est presque une métaphore.

Ainsi la plupart des programmes des TAU appellent de leurs vœux l'implantation de lisières urbaines. Certains projets sont bien avancés comme les aménagements de franges périurbaines dans la plaine de Versailles (à Fontenay-le-Fleury ou à Villepreux), d'autres comme ceux situés en bordure des espaces ouverts de la plaine de Montesson n'en sont qu'à la phase d'étude (figure 3). La finalité de ces aménagements est ambitieuse : d'un côté contenir le front urbain; de l'autre ménager des zones tampons et les aménager (sentiers, mobiliers urbains, pistes cyclables, aires de jeux pour enfants, jardins, plantations...) dans une logique multifonctionnelle. L'idée qui les soustend est que ces interfaces ont vocation à s'épaissir pour devenir des entre-deux d'échange et de circulation tant d'un point de vue social que paysager et écologique, voire alimentaire, et qu'ils joueront ainsi un rôle urbanistique en participant à l'organisation et à la composition de l'espace. Un tel modèle, parce qu'il entend relever de nombreux défis, rencontre un écho très favorable auprès des acteurs institutionnels et associatifs ( $c f$. la consultation du Grand Paris, 2008). Cependant, appliqué à des territoires habités, il se heurte aux pratiques des riverains qui n'ont pas attendu les concepteurs pour aménager et fabriquer entre leurs lieux de vie (ou de travail) et le territoire environnant des limites à leur image ${ }^{12}$. Outre la question des usages sociaux préexistants rarement pris en considération, tant dans la construction théorique de la lisière urbaine que dans sa mise en œuvre, la question foncière (Aragau, 2013) s'affirme aussi comme un verrou puissant à l'application concrète de la figure. Au final, les réalisations effectives se déploient surtout sur du foncier appartenant aux collectivités territoriales et prennent la forme d'aménagement de parcs ou d'espaces publics qui, tout en étant de grande qualité, n'en sont pas pour autant les lieux de dialogue et d'échanges attendus. 
Figure 3. Un projet de paysage de l'agence Folléa Gautier, paysagistes-urbanistes

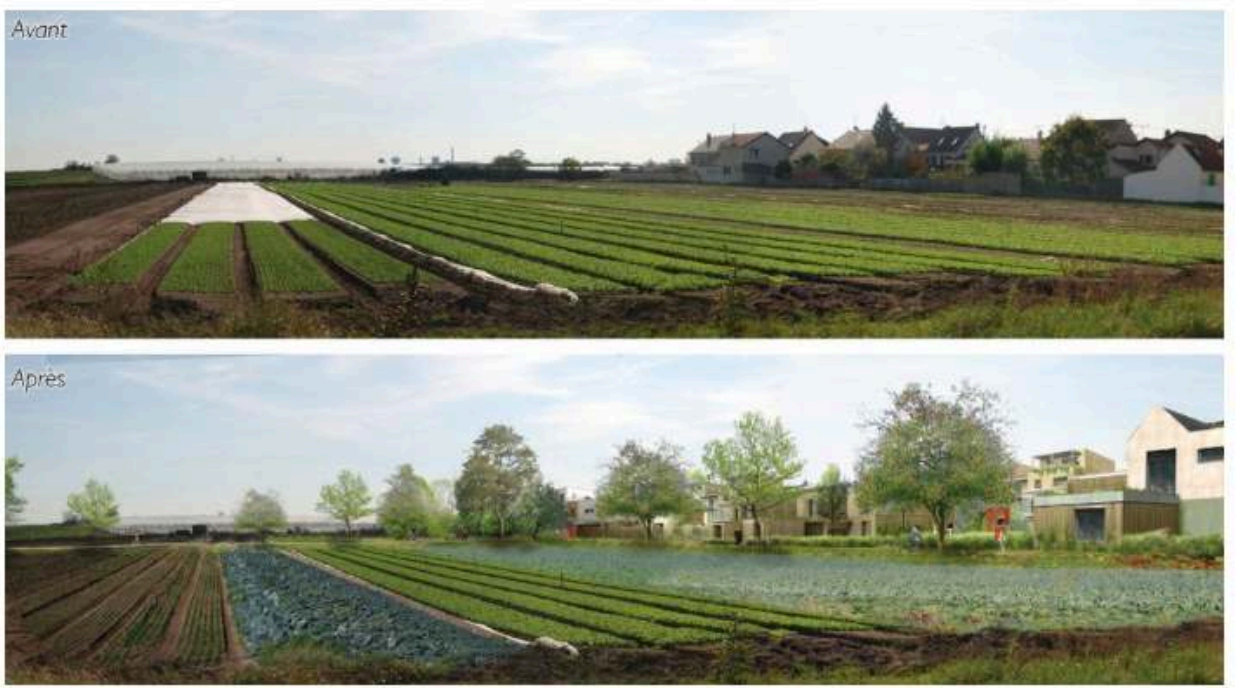

Exemple de mise en place de lisières entre les quartiers résidentiel et la plaine agricole

Pour rapprocher agriculture et bâti dans la plaine de Montesson (2012) : quel statut pour cet « entredeux»?

Source : Agence Folléa Gautier

Lorsque les deux concepts/figures, de lisière et de sentier, donnent lieu à des opérations d'aménagement, leurs effets paysagers sont bien réels : malgré une emprise linéaire et d'épaisseur variable, ils marquent le territoire de façon tangible. De surcroît, en mettant en regard deux mondes aux caractéristiques différentes, l'agricole et l'urbain, tant le sentier que la lisière proposent et véhiculent des images, des représentations de l'un et l'autre des deux univers et de leurs interrelations. Pour autant, sont-elles de nature à modifier la relation paysagère au TAU ? Des enquêtes ${ }^{13}$ menées dans le TAU de Montesson sur les perceptions paysagères des habitants ont souligné le poids des représentations anciennes disqualifiantes (plaine agricole au paysage dégradé et peu accessible) et la difficulté à les faire évoluer malgré un travail sur les lisières véhiculant une volonté d'ouverture. Il semblerait cependant que l'ancienneté de l'installation sur le territoire, la localisation de l'habitation (proximité aux lisières et points de vue sur la plaine) et la fréquentation régulière des espaces agricoles jouent dans les formes d'appropriation habitante, y compris dans le changement de regard ${ }^{14}$. L'efficience de telles constructions sociospatiales qui se veulent vertueuses au regard de questions sociétales - le vivre ensemble, la mixité sociale, la convivialité... - ne va donc pas de soi et reste à démontrer. En effet, peut-on, à travers des arrangements spatiaux, rapprocher culturellement des populations qui le plus souvent s'ignorent, voire entretiennent des relations conflictuelles? 


\section{La question alimentaire saisie par les territoires agriurbains : vers la fabrique de motifs paysagers singuliers?}

\section{La notion de paysage alimentaire ou comment lire un paysage au prisme de la nourriture}

21 Devenue une dimension relativement récente, mais affirmée, de l'agriculture périurbaine, la question alimentaire revêt les atours "d'une mise en proximité " (Guiomar, 2014), laquelle s'impose aujourd'hui comme un principe au fondement des TAU. Longtemps minorée du fait de l'internationalisation croissante des marchés et du développement d'instruments efficaces de distribution dans les pays du Nord, elle revient au premier plan avec les crises alimentaires des dernières années et les revendications fortes d'un «bien manger » qui tient autant du «manger sain » et du "manger juste» que du "manger local». Les short food supply chains sont ainsi plébiscitées: elles placent l'agriculture sous le regard vigilant du consommateur, réduisent les food miles, requièrent une main-d'œuvre importante et contribuent à la création de liens sociaux entre producteurs et consommateurs, tous éléments érigés comme constitutifs d'un développement durable (Deverre et Lamine, 2010 ; Aubry et al., 2012).

Dans les TAU où s'affrontent nécessité de construire, préservation des terres agricoles et injonctions de durabilité, comment s'exprime la préoccupation alimentaire? Façonne-t-elle des paysages spécifiques? Peut-on aller jusqu'à parler d'un paysage alimentaire en train de naître? La notion de paysage alimentaire est récente, utilisée pour la première fois aux États-Unis par Gisèle Yasmmeen en 1996, dans sa thèse de doctorat "Bangkok's foodscape : public eating, gender relations and urban change ${ }^{15}$ »: «Le paysage alimentaire, issu du paysage, est un terme utilisé pour décrire le procédé d'observation d'un lieu à travers le prisme de la nourriture et pour mettre au jour les relations humaines. » Le terme est aujourd'hui largement repris par les chercheurs et les acteurs institutionnels: devenu synonyme d'environnement alimentaire, il «recouvre ce que connaissent les acteurs de l'offre commerciale et de leur environnement alimentaire et la façon dont ils se le représentent.[...] il permet d'éclairer les choix des consommateurs, des producteurs, des acteurs publics en matière alimentaire, de même que les connexions ou déconnexions entre espaces urbains et franges agricoles » (Nikolli et al., 2016) ». Au-delà de cette définition globale, le terme est volontiers appliqué aux terroirs entendus au sens matériel et immatériel, lieux uniques de qualité et de typicité mais aussi de remodelage incessant par des générations d'agriculteurs et donc d'enracinement dans l'histoire: «Le paysage alimentaire est toujours le paysage de quelqu'un, d'un groupe, d'une société, voire d'un État. [...] Il renvoie à l'image que les acteurs se forgent d'eux-mêmes et se réfère, donc, au symbolique et à l'idéologique. [...] Il en devient politique avec leur prise en charge par les tenants $d u$ patrimoine. Il en devient vernaculaire car il manifeste l'appartenance à une communauté de mangeurs dont le terroir est la figure, actuellement, la plus prégnante. » (Fumey, 2014.) On retrouve là la notion de paysage entendue comme une entité relationnelle, celle de l'interaction homme-milieu, envisagée dans sa dimension sociale et culturelle. Ainsi définie, la relation paysagère (sensible et symbolique) au cadre de vie est indissociable des rapports sociaux, de la 
mémoire sociale ou de la connaissance empirique du fonctionnement du milieu (Luginbühl, 2007) ; autrement dit, les pratiques territoriales, les souvenirs, les modes de vie, les croyances interfèrent entre le sujet et l'objet dans la construction, l'appréciation ou la dépréciation du paysage (Fortin, 2014). Tel est le cadre théorique pour l'analyse des paysages alimentaires des TAU développée ci-dessous.

\section{Le retour des paysages nourriciers}

$\mathrm{Au}$ sens premier du terme, le paysage alimentaire ce sont donc des parcelles cultivées pour nourrir la population. Dans une région où la moitié du territoire correspond à des terres agricoles, on pourrait qualifier ces $50 \%$ de paysage alimentaire ou nourricier (DRIAAF, 2012). Mais sur ces $50 \%, 89 \%$ correspondent à des grandes cultures (céréales et oléoprotéagineux) s'inscrivant dans un marché global (européen, mondial) et 7,6 \% à des jachères.

Lorsque ces vastes superficies n'ont pas un objectif énergétique, leur consommation humaine est lointaine (filières de commercialisation et de transformation complexes et longues) et parfois indirecte (consommation animale). Bien que destinées pour une grande part à l'alimentation de l'homme, elles ne sont pas perçues spontanément comme ayant une vocation alimentaire par les non-agriculteurs; ceux-ci la réservent plutôt pour les surfaces maraîchères ou fruitières alors même qu'en Île-de-France elles n'occupent qu'à peine $3 \%$ du territoire agricole (soit $0,8 \%$ en légumes, $0,5 \%$ en pommes de terre, $0,2 \%$ en cultures fruitières, $1,3 \%$ en divers, $0,6 \%$ en prairies artificielles). Dans les TAU, de telles représentations sociales valorisant les nourritures jardinières ${ }^{16}$ sont assez communes, elles dessinent autour de quelques denrées un paysage alimentaire, qualifié de vivrier ou de nourricier du fait de l'absence d'intermédiaire entre le champ et l'assiette (la proximité est alors une valeur) (Allemand et Heurgon, 2016). Elles se heurtent à celles des agriculteurs pour qui les espaces de grandes cultures, dont la progression a été encouragée par la politique agricole commune (PAC, 1962) afin de garantir la sécurité et l'indépendance alimentaires de l'Europe (traité de Rome, 1957), participent tout autant du paysage alimentaire ${ }^{17}$.

Le recul des cultures spéciales (légumes, fruits, fleurs) a entraîné dès l'entre-deuxguerres, dans un contexte d'ouverture des marchés agricoles et de spécialisation des régions, la rétraction de la ceinture maraîchère et horticole francilienne située dans la première couronne, puis sa quasi-disparition au profit de l'urbanisation et des grandes cultures (Poulot et Rouyres, 2000). Jusque dans les années 1990, alors que les logiques de mondialisation économique, culturelle, politique s'imposent, ce retrait n'est pas pointé comme un problème et il est accompagné du transfert des cultures spéciales toujours plus loin en Île-de-France : il en est ainsi des ateliers maraîchers de plein champ qui se créent dans certaines exploitations de grande culture, par exemple autour de Fontainebleau (77) ou encore à Gally (78) où la première ferme de cueillette voit le jour en RIF dans les années 1970 ; il en est de même des quelques lotissements maraîchers créés loin du front urbain, comme le "domaine agrotouristique " de Périgny (94) réalisé en 1977 pour accueillir des horticulteurs expulsés de Créteil (Poulot, 2011). Mais depuis le début des années 2000, la dynamique s'est pour ainsi inversée et les initiatives se multiplient pour redévelopper sur des territoires situés dans un rayon de 10 à $30 \mathrm{~km}$ du centre de Paris (Ceinture verte d'île-de-France ${ }^{18}$ ) une 
agriculture perçue comme nourricière. Les TAU n'en sont qu'une expression parmi d'autres car sur la totalité du territoire francilien sont désormais repérables des réorientations de grosses exploitations ou des installations ${ }^{19}$ sur des microfermes, pratiquant des cultures spéciales associées, par les consommateurs, à l'alimentation (maraîchage, arboriculture fruitière, élevages...). Le choix des productions dépend des perceptions que les agriculteurs se font de la demande locale, laquelle objectivement variera en fonction des caractéristiques sociales, économiques et culturelles des populations habitant le territoire (enquête auprès des exploitants : Le Plessis-Gassot, 95). On retrouve ici la définition matérielle du paysage alimentaire et une cartographie fine des TAU montrerait les évolutions en cours d'autant que les jardins, autres formes de paysages alimentaires vivriers, y connaissent un fort développement (Faramond et al., 2015).

\section{Des paysages publicisés et publicitaires}

Le paysage alimentaire se repère également dans les modes d'organisation spatiale des bâtiments d'exploitation et des parcellaires pensés pour accueillir les consommateurs devenus acheteurs-cueilleurs. Les fermes de cueillette de Gally (78) et de Compans (77) en sont des illustrations commodes pour la démonstration, le tout dans des environnements sonores et de communication qui rappellent la situation métropolitaine (proximité d'échangeurs et d'axes autoroutiers à forts débits, ballet d'avions dans le triangle de Roissy). Parcourir leurs sites s'apparente à déambuler entre les rayons d'un supermarché, la cagette ou la brouette s'étant substituées au Caddie à remplir. Après consultation à l'entrée d'un vaste panneau indiquant les produits disponibles et leurs prix, à vous de choisir les légumes et les fruits que vous recherchez. Le site est aménagé de façon structurée, rectiligne et sans surprise : chaque carré est identifié à partir du produit qu'il propose, production rappelée sur un panneau installé en tête de parcelle (figure 4); après avoir récolté fruits et légumes, et peut-être pour finir un petit bouquet de fleurs, le consommateur passe à la caisse pour payer. S'il n'a pas le temps de flâner et de s'adonner à l'acte de cueillir, dans la boutique (P'tit Marché gourmand à Compans) qui se trouve à l'entrée (ou à la sortie) (figure 5), fruits et légumes ont été ramassés pour lui, si bien que son panier sera vite plein ; il peut même commander et payer en ligne, puis passer en voiture prendre sa commande (Drive de Compans), « cela ne vous prendra que 5 minutes pour charger la commande dans votre coffre ». À Gally, le visiteur arpentera un vaste magasin articulant plusieurs univers (jardinerie, animalerie, marché des terroirs...), en libre-service pour la plupart. Finalement, l'analogie avec la grande surface est bien opératoire. 
Figure 4. Un parcellaire organisé pour la vente et repéré par des panneaux disposés sur des gondoles (Les 2 cueillettes de Compans, 95)

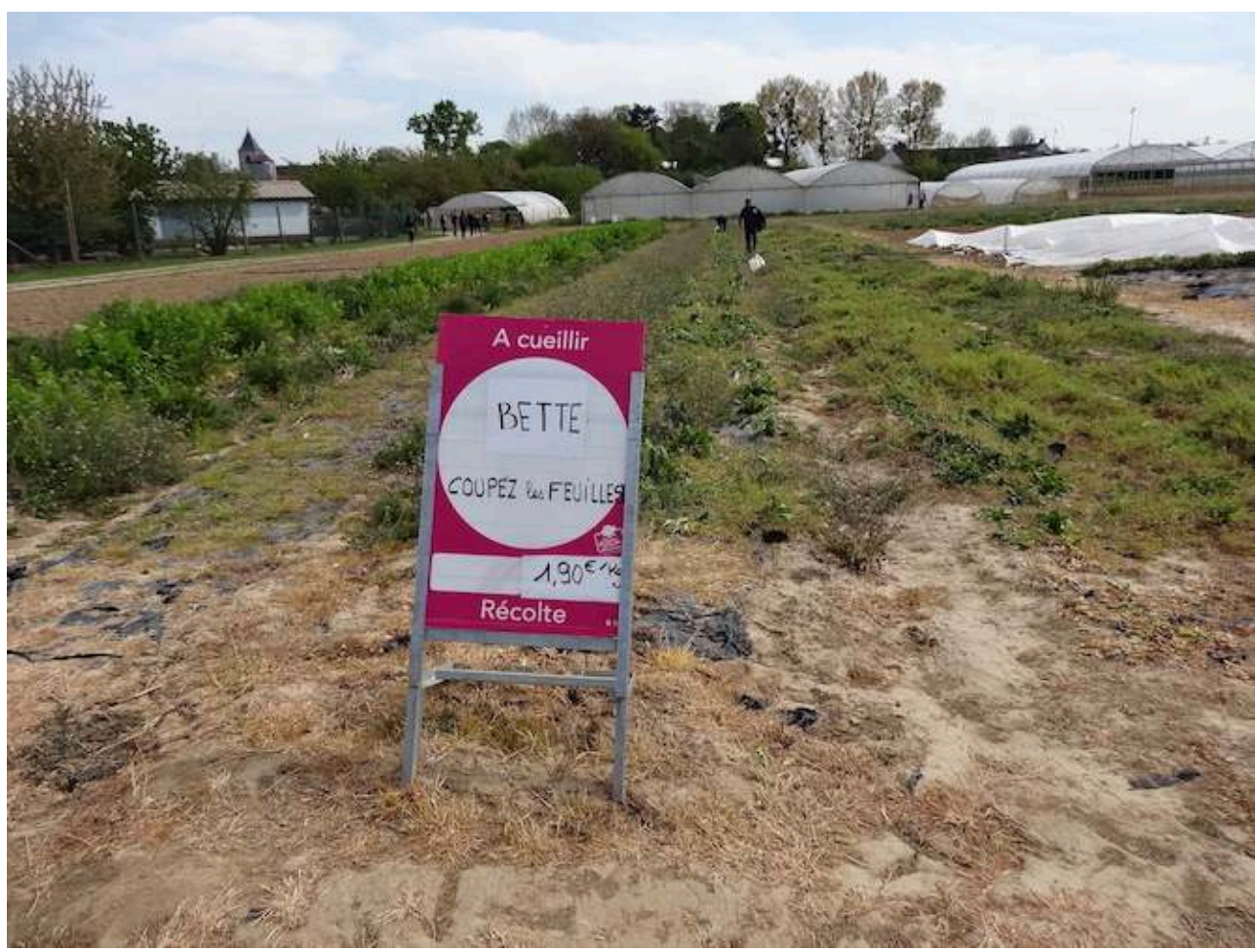

On notera la dimension pédagogique du message à l'adresse des cueilleurs.

Source : Monique Toublanc, 2017.

Figure 5. Quand l'acte de cueillir devient un simple acte d'achat (le P'tit Marché Gourmand à Compans, 95)

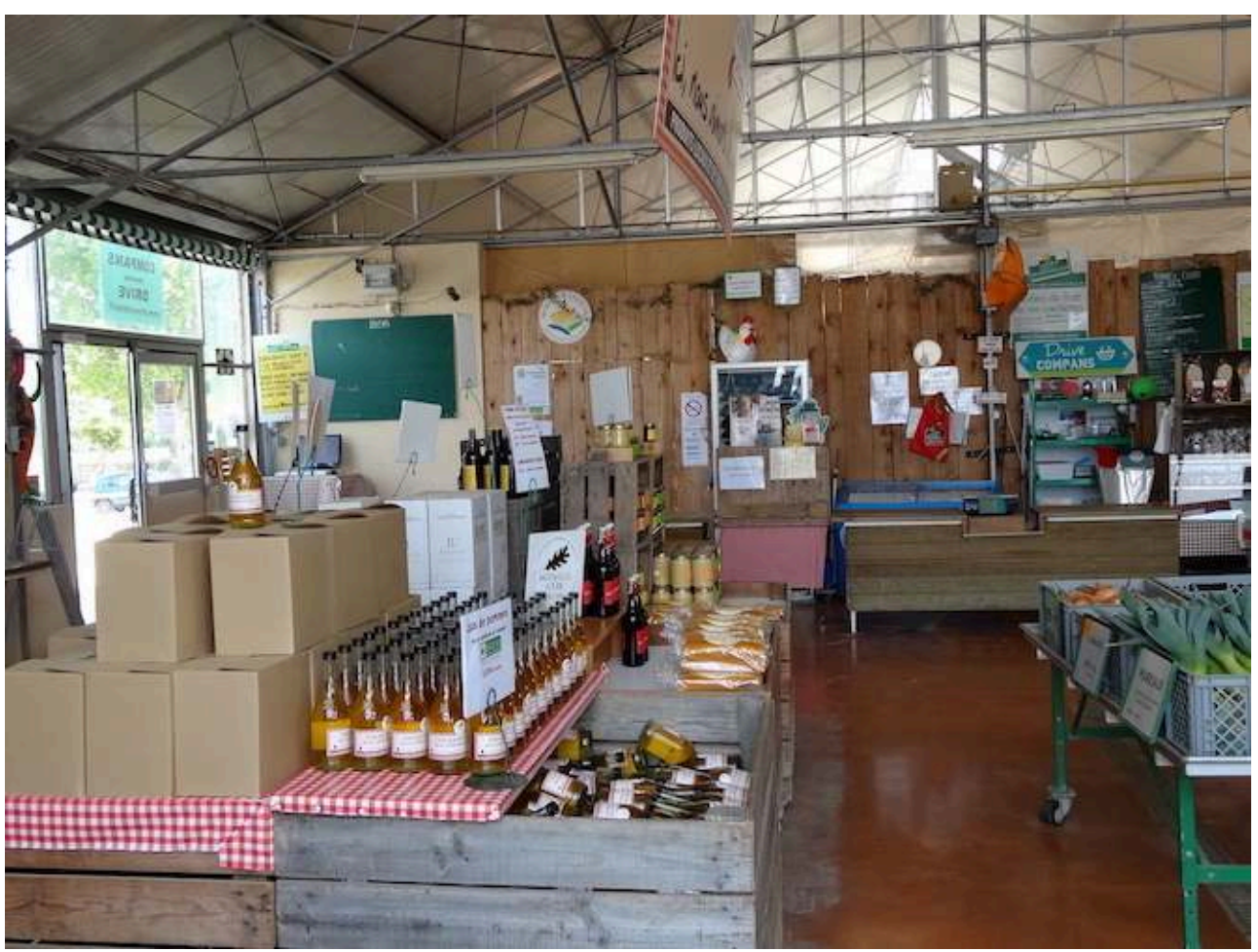

Source : Monique Toublanc, 2017. 

enfants si les acheteurs viennent en famille (aires de jeux ou panneaux ludiques pour se faire prendre en photo...) participent aussi de ce paysage alimentaire. Et surtout, la plupart des fermes ouvertes au public (cueillette ou uniquement vente directe) s'attachent à correspondre à une certaine image de ferme, plutôt la ferme à l'ancienne telle que la publicité la vante, voire telle que nombre d'urbains se l'imaginent comme l'ont montré les enquêtes en TAU mais aussi dans les parcs naturels régionaux franciliens (Raymond, 2004). Tous les attributs que l'imaginaire social, la mémoire collective associent à l'agriculture s'y retrouvent, en particulier les animaux de la ferme (basse-cour, cochon, âne, cheval...) devenus éléments de décor - car il s'agit rarement de fermes d'élevage - à moins qu'ils ne soient des supports aux activités pédagogiques ${ }^{20}$ développées par les agriculteurs à l'adresse des visiteurs.

Outre ces aménagements spécifiques du territoire de l'exploitation agricole, le paysage alimentaire se lit (et s'exprime) également dans les dispositifs spatiaux visibles de l'espace public ou installés sur celui-ci dont la finalité est de rapprocher les urbains, consommateurs potentiels habitant dans les TAU, et les agriculteurs qui produisent sur ces mêmes territoires. Ce sont les multiples panneaux de grande taille annonçant les ventes et les cueillettes à la ferme (figure 6); ce sont parfois des distributeurs automatiques installés sur le bord de la route à proximité de l'exploitation (figure 7) ou bien des boutiques installées à l'entrée de la ferme ; ce sont également des stands isolés ou des marchés de producteurs implantés sur la place des villages ou d'autres sites stratégiques faciles d'accès... De tels dispositifs spatiaux sont présentés comme participant d'une dynamique de rapprochement producteurs/consommateurs, ville/ agriculture: modes de commercialisation en circuits courts mais aussi proximité sociale, lieux de rencontres et de sociabilité. 
Figure 6. Un paysage commercial hérissé de panneaux signalétiques appelant le consommateur (Les 2 cueillettes de Compans, 95)

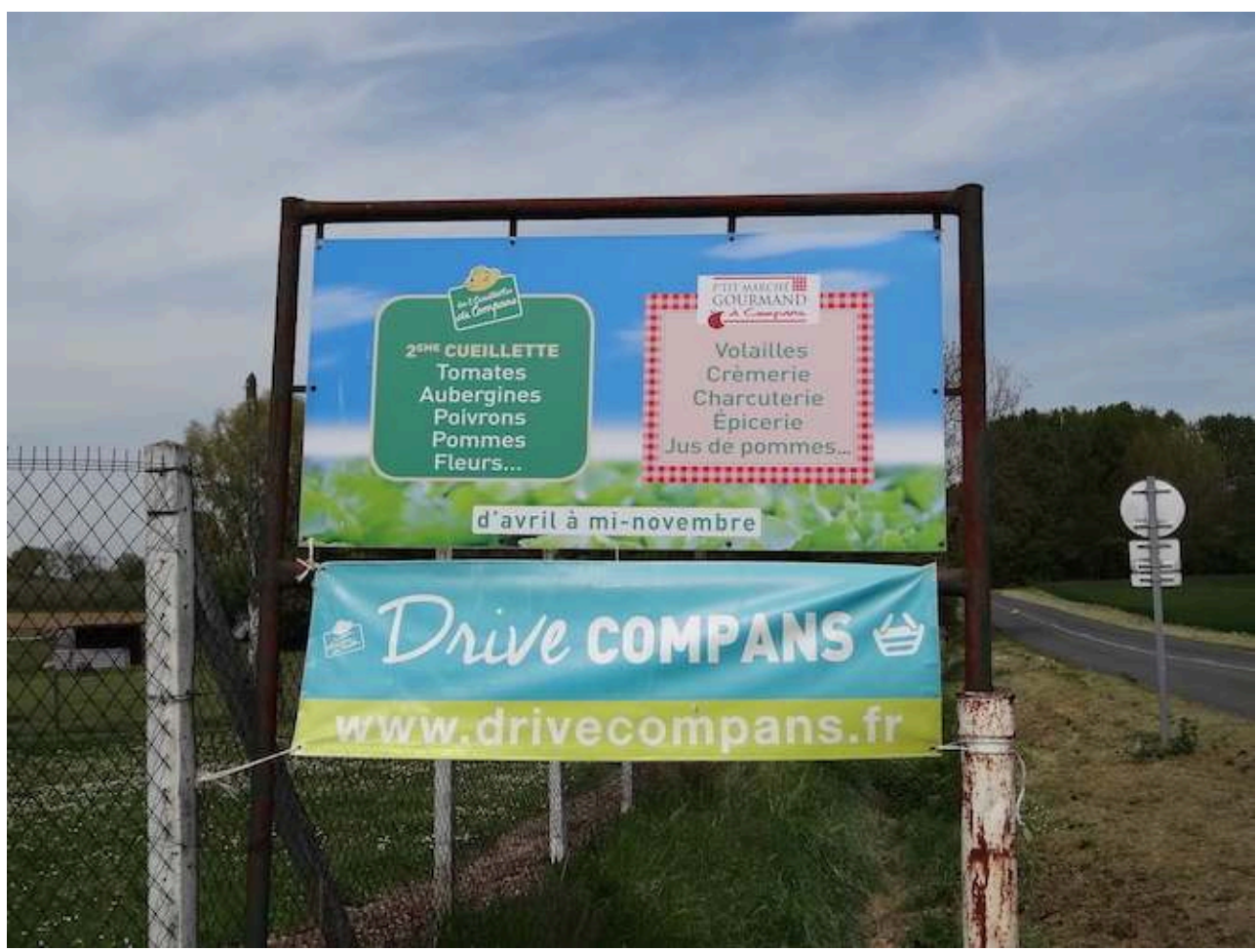

Source : Monique Toublanc, 2017

Figure 7. Un distributeur automatique de produits frais de la ferme (ouvert $7 \mathrm{~J} / 7 \mathrm{~J}$ de $8 \mathrm{~h}$ à $20 \mathrm{~h}$ ) ou l'impossible recherche de lien social (la ferme du Plessis-Gassot, 95)

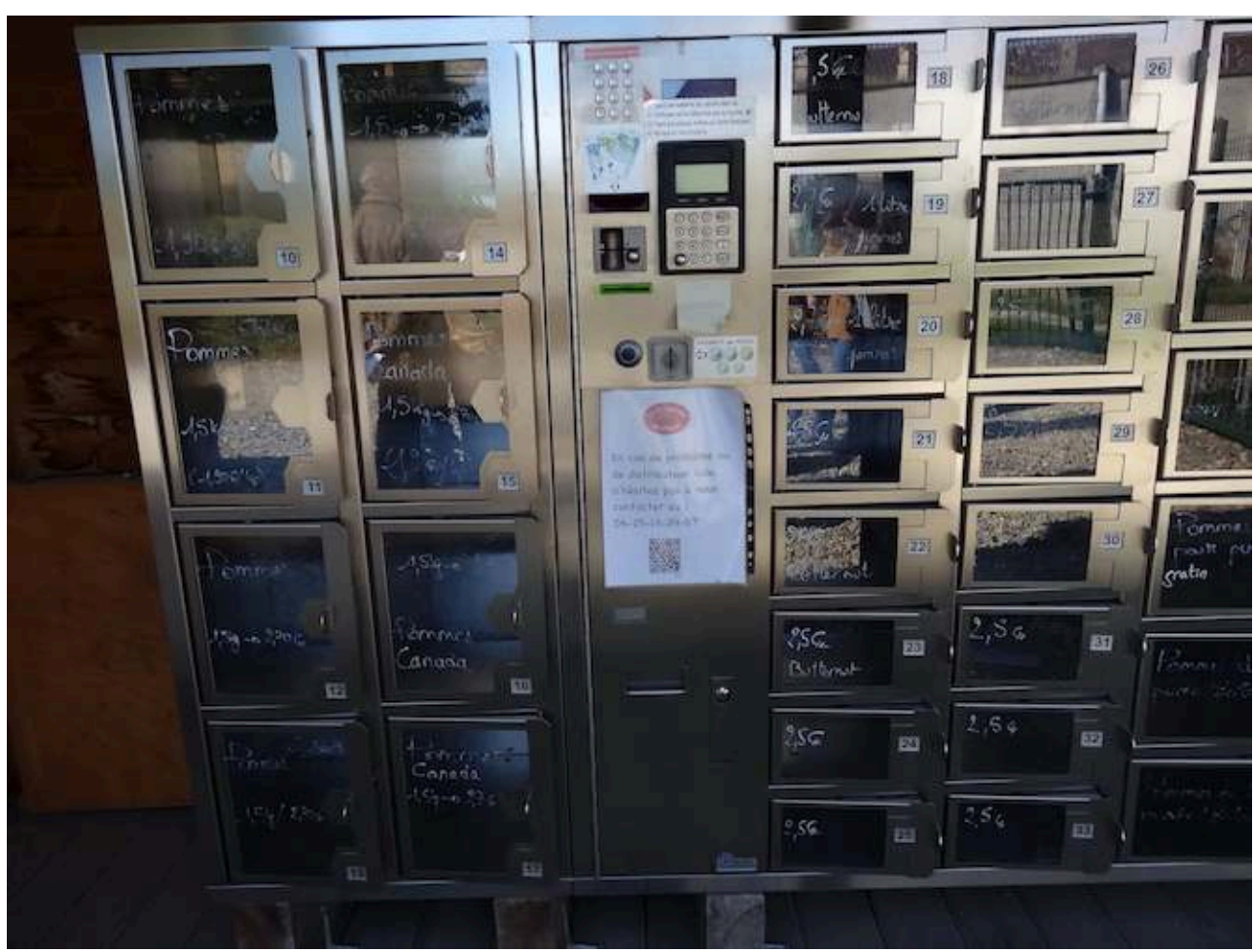

Source : Monique Toublanc, 2017 
Le paysage alimentaire en construction dans les TAU est ainsi ponctué de signes, de marques à l'image des paysages commerciaux: il s'agit d'attirer le consommateur en vantant des manières de faire différentes (produire et vendre local), de donner à voir certains actes de l'agriculture - surtout le produit final -, d'intégrer l'agriculture dans le bassin de vie du consommateur-habitant et partant, de tisser des liens de reconnaissance pour aller vers une appropriation. Le phénomène est relativement récent et il est difficile d'en mesurer l'impact réel sur les habitants-consommateurscitoyens. Toutefois, la rencontre n'est pas toujours au rendez-vous, ni même désirée : "Je veux bien faire de la vente directe mais je ne veux pas voir les clients." (Agriculteur installé dans le Val-d'Oise.) La contradiction est résolue par la mise en place de distributeurs automatiques qui établissent une mise à distance mais n'en sont pas moins appréciés par les consommateurs (Faramond et al., 2015). On peut également s'interroger sur les mises en scène dans les cueillettes franciliennes: en effet, si l'acte de cueillir instaure un rapport physique à la terre et au produit, structurant la relation paysagère de l'acheteur avec le territoire agricole (Rue, 2015), les aménagements franciliens ${ }^{21}$ - notamment du fait du nombre de consommateurs visés - sont assez élaborés. De fait, ils ne montrent guère l'exploitation agricole comme lieu de production, non plus que les manières de produire. Le visiteur est tenu à distance ; dans certains cas même, les aménagements étant au service d'activités pédagogiques, sociales, festives... il n'est plus accueilli par l'agriculteur lui-même mais par des personnes recrutées pour leurs compétences en animation ou en communication.

À partir du paysage alimentaire, les TAU relayés par de nombreux agriculteurs surfent enfin sur un slogan «Produire et consommer localement, c'est préserver notre environnement»: ce dernier fait de la proximité une vertu sur le plan écologique (qualité de l'environnement, réduction des food miles...) et sur le plan sanitaire et gustatif (qualité de la denrée consommée). Les circuits courts, la vente directe... seraient ainsi les garants d'une agriculture plus vertueuse, plus attentive à l'environnement à rebours de la période productiviste. Cette croyance qui reste scientifiquement à démontrer tant les paramètres à considérer pour évaluer les services écosystémiques sont multiples (Huang et al., 2015) est largement diffusée dans les TAU : elle vient nourrir une image positive de l'agriculture et, par ricochet, celle d'un paysage amène, à laquelle les agriculteurs sont, d'une certaine manière, conviés à adosser l'image de leur ferme pour œuvrer à la connexion attendue.

31 L'idée de proximité tend enfin à masquer les aspects techniques des exploitations agricoles qui en Île-de-France peinent à modifier leurs pratiques et leurs systèmes de production vers une meilleure prise en compte des questions environnementales. Si les microfermes et les nouveaux installés dans le cadre de contrats de fermage à long terme avec la Région doivent obligatoirement adopter un mode de production biologique, nombreux sont ceux qui hésitent à se lancer dans cette transformation, souvent complexe à mener et sans réelle garantie (Petit, 2013) et s'orientent plutôt vers une agriculture raisonnée (DRIAAF, 2014). Les TAU n'échappent pas à cette tendance même si les conversions à l'agriculture biologique paraissent plus nombreuses que dans le reste de l'île-de-France (Gautron, 2017). Surtout, les agriculteurs engagés dans la vente à la ferme que nous avons pu rencontrer s'interrogent sur l'intérêt de communiquer sur le fonctionnement technique de leur structure - certains s'y refusent arguant que cela n'intéresse pas le client - puisque les demandes des consommateurs- 
habitants ont trait essentiellement à la proximité, ce que l'ouverture de l'exploitation garantit bel et bien.

\section{Conclusion}

Les TAU apparaissent comme des lieux clefs où s'élabore une grammaire paysagère d'abord autour de la seule agriculture, corrélée ensuite à la question alimentaire. Ils font figure en ce sens de terrains d'expérimentation pour la métropole francilienne dont le projet s'est transformé au fil du temps. Les premiers schémas régionaux, jusqu'en 1990, ont surtout œuvré à protéger les espaces forestiers, les espaces agricoles ouverts ayant quant à eux vocation à accueillir la périurbanisation, habitats et infrastructures confondus. Les deux derniers, en revanche, et notamment le SDRIF de 2013, accordent une importance nouvelle aux espaces agricoles en tant qu'« élément incontournable du développement régional » et à l'agriculture reconnue en premier lieu pour "sa production importante", avant sa "contribution au cadre de vie " et " son rôle dans la fonctionnalité des milieux " ${ }^{22}$. Si en 1994, les préconisations visaient à maintenir et à promouvoir le paysage agricole déjà là, sans attentes précises sur ses propriétés mais en tant qu'élément constitutif d'une certaine organisation territoriale, voire comme espace réversible où tout restait possible, $\mathrm{y}$ compris la construction de bâti (Toublanc et Bonin, 2012), l'agriculture souhaitée dans le SDRIF de 2013 est caractérisée et placée notamment sous le signe de la proximité. À la faveur de l'affirmation du débat alimentaire sur la scène publique, ce type d'agriculture, et les paysages qu'il façonne sont perçus comme les ingrédients substantiels d'une métropole durable par les acteurs institutionnels et politiques. Conforter et restaurer une agriculture de proximité est un axe décliné à l'échelle de la RIF au nom d'une stratégie alimentaire (Programme alimentaire régional, 2012), d'où le soutien régional réaffirmé aux TAU, mais aussi par conséquent à l'agriculture intra-urbaine, laquelle est finalement davantage médiatisée car perçue dans le contexte européen comme plus novatrice que l'agriculture périurbaine ${ }^{23}$. Ces derniers participent ainsi, bien au-delà de leurs stricts périmètres, du "projet de paysage " porté aujourd'hui par les instances régionales et il est significatif que sur la douzaine de programmes en cours ou en expérimentation, plus de la moitié ait pour accroche les reliques de l'ancienne ceinture maraîchère francilienne : un dispositif défini dès 1983 qui a toujours peiné à s'affirmer et qui semble aujourd'hui connaître une nouvelle jeunesse, même si à la figure de la ceinture s'est substituée celle d'archipels, d'espaces non bâtis - agricoles, boisés ou verts (parcs, ripisylves...) - en réseau (Agrige, 2015).

Dans cette forme urbaine en devenir qui reconduit une logique concentrique (une couronne de TAU au contact du front urbain et une couronne de PNR sur les franges de la Région), les TAU témoignent à leur manière d'une certaine évolution du métier d'agriculteur dans une société où la population vivant dans un milieu défini comme urbain n'a cessé d'augmenter ( $80 \%$ en France en 2017). À travers l'émergence de l'agriurbanisme dans les années 1990, les agriculteurs se sont vus chargés par la collectivité de prendre part à la construction de la ville, et plus largement à l'aménagement de l'espace : un changement à l'œuvre en milieu rural dès la fin de la décennie 1980 quand s'impose la figure de l'agriculteur, jardinier de la nature face au développement des friches. Toujours conviés à produire des denrées, les agriculteurs sont alors appelés à assurer d'autres missions comme produire des espaces ouverts 
ayant une finalité récréative, créer du sens, de l'harmonie, des paysages, renouer avec la nature, être les garants d'une gestion durable des ressources naturelles... Pour une profession dont la raison d'être et la fonction essentielle sont historiquement de cultiver la terre pour produire de quoi se nourrir et nourrir ses pairs, cela suppose un retournement instrumental auquel de nombreux agriculteurs ont résisté, voyant dans cette injonction une perte de sens et d'utilité sociale de leur activitée ${ }^{24}$. Qui eut cru que 20 ans plus tard la fonction alimentaire et donc la production agricole reviendraient sur le devant de la scène en particulier aux abords et au sein des grandes villes? Ce revirement ne manque pas d'interroger et en cela la dynamique des TAU est édifiante.

\section{BIBLIOGRAPHIE}

Allemand, S., Heurgon, E. (coord.), Nourritures jardinières dans les sociétés urbanisées, colloque de Cerisy, Paris, Hermann, 2016, 250 p.

Aragau, C., 2013, « L'agriculture et le projet urbain : exemples d'aménagements fonciers en plaine de Versailles ", dans Poulot, M. (coord.), Agriculteurs pour la ville, agriculteurs dans la ville: acteurs, pratiques et enjeux, BAGF, $\mathrm{n}^{\circ} 3$, p. 377-395.

Aubry, C., Kebir, L., Pasquier, « Le raccourcissement des circuits alimentaires : une nouvelle ruralité en périphérie des villes ? (étude de cas en Île-de-France) ", dans Papy, F., Mathieu, N., Ferault, C. (dir.), Nouveaux Rapports à la nature dans les campagnes, Versailles, Éditions Quae, 2012, p. 13-22.

Aubry, C., « Les agricultures urbaines et les questionnements de la recherche », Pour, n²24, 2014, p. 35-49.

Bataille, S., « Conception d'un sentier d'interprétation agricole en zone périurbaine », mémoire de DESS, université François Rabelais de Tours, 2002, 108 p.

Bergerie nationale, « Les programmes agriurbains d'île-de-France. État des lieux, bilan et perspectives en $2016 », 2016,112$ p.

Berger, M., Les Périurbains de Paris. De la ville dense à la métropole éclatée, Paris, CNRS Éditions, 2004, $317 \mathrm{p}$.

Béringuier, P. et al., « Les paysages des franges urbaines, transitions ou parois de verre ? » dans Luginbühl, Y. (dir.), Biodiversité, Paysage et Cadre de vie, Paris, Victoires Éditions, 2015, p. 71-89.

Bernadet, S., Cheveau, C., Grenier, A. et Le Cam, M., Une terre de grande culture aux portes de Paris : plus de cinquante ans de productions agricoles franciliennes, Paris, SRISE Île-de-France, 2016, $124 \mathrm{p}$.

Berque, $\mathrm{A}$, « La transition paysagère comme hypothèse de projection pour l'avenir de la nature " dans Roger, A., Guéry, F. (dir.), Maîtres \& protecteurs de la nature, Paris, Champ Vallon, 1991, p. 217238.

Berque, A, Médiance : de milieux en paysage (1990), Paris, Belin, 2000, 161 p.

Biasi, L. de et Stephan, J.-M. (dir.), Atlas Rural et Agricole de l'île-de-France, 2004, Paris, DRIAAF-

IAURIF, $180 \mathrm{p}$. 
Bonin, S., "The concept of urban lisiere and its application on Reunion Island ", dans Lambertini, A. (dir.), The Role of Open Spaces in the Transformation of Urban Spaces, II Faro, Editrice Compositori, 2013, p. 211-226.

Brand, C. et al., Construire des politiques alimentaires urbaines, Versailles, Éditions Quae, 2017, 160 p.

Chalas, Y., « La ville-nature contemporaine. La demande habitante à l'Isle-d'Abeau », Annales de la recherche urbaine, $\mathrm{n}^{\circ} 98,2005$, p. 43-49.

Deverre, C., Lamine, C. « Les systèmes agroalimentaires alternatifs, une revue de travaux anglophones en sciences sociales », Économie rurale, $\mathrm{n}^{\circ}$ 317, 2010, p. 57-73.

Donadieu, P., Campagnes urbaines, Arles/Versailles, Actes Sud/École nationale supérieure de paysage, 1998, $219 \mathrm{p}$.

Donadieu, P., Mazas, E., Des mots de paysage et de jardin, Dijon, Educagri, 2002, 316 p.

Faramond, J. de et al., « Habiter l'agri-urbain ou l'exemple de la plaine de Montesson », université de Paris Nanterre, commande PSDR Agrige, 2015, 122 p.

Fortin, M.-J., « Les paysages de la transition énergétique : une perspective politique », Projets de paysage, $\mathrm{n}^{\circ}$ 10, décembre 2014, URL : http://www.projetsdepaysage.fr/fr/

les_paysages_de_la_transition_energetique_une_perspective_politique

Fumey, G., « Paysages à boire et manger », Les Carnets du paysage, Arles/Versailles, Actes SudENSP, 2014, $\mathrm{n}^{\circ} 25$.

Gautron, V., «Quelle place pour les agriculteurs au sein d'un territoire sous emprise urbaine à travers le concept de mode d'habiter : le cas du territoire agriurbain de Seine-Aval », mémoire de master 2, PSDR Agrige, 2017, $136 \mathrm{p}$.

Genest, A., « Les sentiers d'interprétation en région Île-de-France. Un outil d'échanges en France périurbaine ? ", mémoire de master DPLG 3, sous la direction de Monique Toublanc, ENSP, 2016, 97

Guiomar, X., « La mise en proximité de l'agriculture (péri)urbaine par les collectivités. Une reconstruction en trois temps, trois mouvements ", Pour, 2014, n²24, p. 415-426.

Guiomar, X., «Les politiques et les lois visant à développer l'agriculture (péri)urbaine française », Demeter, 2013, p. 157-180.

Guiomar, X., «Éveiller le regard du marcheur sur l'agriculture : les sentiers d'interprétation agricole en Île-de-France ", Pour, 2010, n²05-206, 10 p.

Huang, J., Tichit, M., Poulot, M., Darly, S., Petit, C., Aubry, C., « Comparative review of multifunctionality and ecosystem services in sustainable agriculture », Journal of Environmental Management, vol. 149, 2015, p. 138-147.

Lardon, S., Loudiyi, S. (dir.), « Agriculture et alimentation urbaines : entre politiques publiques et initiatives locales », Géocarrefour, 2014, 89/1-2, URL : http://journals.openedition.org/ geocarrefour/9362.

Luginbühl, Y., « Le paysage rural. La couleur de l'agricole, la saveur de l'agricole mais que reste-til de l'agricole ", Études rurales, $n^{\text {os }} 121-124,1991$, p. 27-44.

Luginbühl, Y., « La place de l'ordinaire dans la question du paysage », Cosmopolitiques, 15, 2007, p. 173-177.

Luginbühl, Y., programme de recherche « Politiques publiques et paysages. Analyse, évaluation, comparaisons. Synthèse des résultats scientifiques », MEDDE-DGALN-CEMAGREF, 2004, 22 p. 
Mendras, H., La Fin des paysans (1967), Arles, Actes sud, coll. « Babel », 1992.

Nikolli, A., Le Gall, J. et Laval, M., « Les marges sociales et les franges agricoles se tournent-elles le dos ? », Projets de paysage, n 13, janvier 2016, URL : http://www.projetsdepaysage.fr/fr/ les_marges_sociales_et_les_franges_agricoles_se_tournent_elles_le_dos_\#

Petit, C., « Transitions des exploitations agricoles vers l'agriculture biologique dans un territoire : approche par les interactions entre systèmes techniques et de commercialisation. Application aux aires d'alimentation de captage en Île-de-France ", thèse de doctorat AgroParisTech sous la direction Christine Aubry, 2013, 392 p.

Phlipponneau, M., La Vie rurale de la banlieue parisienne, étude de géographie humaine, Paris, Éditions Colin, 1956, 593 p.

Poulot, M., « L'invention de l'agri-urbain en Île-de-France. Quand la ville se repense aussi autour de l'agriculture », Géocarrefour, 89/1-2, 2014, p. 11-19, URL : http://journals.openedition.org/ geocarrefour/9363.

Poulot, M., « Du vert dans le périurbain », EspacesTemps.net, 6 mai 2013, URL : http:// www.espacestemps.net/articles/du-vert-dans-le-periurbain-les-espaces-ouverts-unehybridation-de-lespace-public/

Poulot, M., « Des arrangements autour de l'agriculture en périurbain : du lotissement agricole au projet de territoire », VertigO, vol. 11, n² 2, septembre 2011, URL : http://vertigo.revues.org/ 11188.

Poulot, M., « Des territoires de projet en périurbain : les programmes agri-urbains », Historiens \& Géographes, 403, 2008, p. 159-172.

Poulot, M. et Rouyres, T., « La ceinture maraîchère et horticole francilienne entre production économique et production de paysage ", Méditerranée, vol. 95, n 3, 2000, p. 51-57.

Raymond, R., « La nature à la campagne : identification sociale et argument pour la gestion d'un territoire rural partagé : l'exemple du Vexin français ", thèse de doctorat en géographie, université de Paris 1, 2004559 p.

Rue, M., « Raviver l'hospitalité des pentes du pays Thiernois. Être accueilllant », TPFE, ENSP Versailles, 2015, 180 p.

Salomon Cavin, J., « La ville au secours de la campagne, une politique urbaine pour protéger l'Angleterre rurale », Espaces et Sociétés, n 126, 2006/3, p. 141-158.

SDRIF, « Île-de-France 2030. Évaluation environnementale. Projet de schéma directeur de la Région Île-de-France », 2012, 112 p.

Steel, C., Ville affamée. Comment l'alimentation façonne nos vies (2008), traduit de l'anglais par M. Bouvier, Paris, Rue de l'échiquier, 2016, 448 p.

Tilden, F., Bomar, M., Craig, B., Interpreting our heritage (1957), Chapel Hill, University of North Carolina Press, 2008, 212 p.

Toublanc, T., Bonin, S., Dérioz, P. (dir), dossier thématique « Paysages des franges urbaines en projet ", Projets de Paysage, janvier 2016, n 13, URL : http://www.projetsdepaysage.fr/ n_13_paysages_des_franges_urbaines_en_projet

Toublanc, T. et Bonin, S., « Planifier les trames vertes dans les aires urbaines : une alliance à trouver entre paysagisme et écologie ", Développement durable et territoires, vol. 3, n² 2, juillet 2012, URL : http://developpementdurable.revues.org/9347 
Vidal, R. et Fleury, A., « De la ville nature à la ville agriculture : le projet agriurbain », Anthos, $\mathrm{n}^{\circ} 3$, 2007, p. 59-60.

Yasmmeen, G., Bangkok's foodscape: public eating, gender relations and urban change, Banglamung, White lotus press, 2006.

\section{NOTES}

1. À une échelle régionale, les zones naturelles d'équilibre (1976), la ceinture verte (1983), ou à une échelle plus locale, le premier programme du plateau de Saclay en 1976, et la création de lotissements agricoles comme ceux de Périgny-sur-Yerres (années 1970) ou en regard de la ville nouvelle de Cergy (début des années 1980) sont portés par une volonté forte de conserver des espaces agricoles : ces espaces ainsi définis et les procédures qui les accompagnent préfigurent d'une certaine manière les programmes agriurbains.

2. Cf. Télérama, « Comment la France est devenue moche? », février 2010.

3. Agrige (2016-2020) est porté conjointement par AgroParistech, l'université de Paris Nanterre et l'École nationale supérieure de paysage de Versailles ainsi que le CAUE de l'Essonne en partenariat avec les six territoires agriurbains analysés : APPVPA (plaine de Versailles), Terres et Cités (plateau de Saclay), ADADSA (Seine Aval), Plaine d'avenir 78 (Montesson), Triangle vert (plateau de Marcoussis), communauté d'agglomération du Val d'Orge (Brétigny), conseil départemental de Seine-et-Marne (notamment pour le Grand Roissy).

4. «Paysages des franges périurbaines. Représentations, indicateurs, outils (PFP-RIO)», programme de recherche PDD2, ministère de l'Écologie, 2010-2014; «Le périurbain ouest francilien: de l'hybridation à l'intensité ?", programme Puca «Du périurbain à l'urbain ", 2011-2014 ; cf. Poulot, M., Aragau, C., Berger, M., Rougé, L., Mettetal, L. (éd.), 2014, «Les territoires périurbains : de l'hybridation à l'intensité ", Puca, Lavue- Mosaïques, Prodig, ESO, IAU, $320 \mathrm{p}$.

5. Le programme Agrige a donné lieu à plusieurs mémoires de master 1 ou 2 utilisés dans cet article : Diallo, A., «Étude des outils de sauvegarde et procédures foncières dans le périurbain : l'exemple du Triangle vert ", master 1, 2016, 94 p. ; Cotigny, J., " Synthèse des outils de protection $\mathrm{du}$ foncier dans les TAU en : on : l'exemple de Vernouillet et du plateau de Saclay », master 1, 2016, 92 p. ; Gautron, V., « Quelle place pour les agriculteurs au sein d'un territoire sous emprise urbaine à travers le concept de mode d'habiter : le cas du territoire agriurbain de Seine-Aval», master 2, 2017, 136.

6. Elle reconduit dans certaines formes la ceinture maraîchère mise en place au XIXe siècle dans la première couronne de la RIF (Phlipponneau, 1956).

7. Genest, A., «Les sentiers d'interprétation en région île-de-France. Un outil d'échanges en frange périurbaine ? ", ENSP, mémoire de master DPLG 3, sous la direction de MoniqueToublanc, 2016, $97 \mathrm{p}$.

8. Cf. figure 2, «Le paysage nourricier du domaine de Saint-Leu ». Les panneaux mettent l'accent sur la fraîcheur des produits (circuit court « de la graine... à l'assiette ») et sur l'emploi raisonné des intrants.

9. La culture de la menthe poivrée, la seule qui soit officinale et dont la réputation est mondiale, a valu à Milly-la-Forêt cette distinction. Héritiers d'une tradition médiévale, les quelques producteurs actuels de plantes médicinales - plus de 50 en 1950 - fournissent les grands laboratoires pharmaceutiques.

10. Aux producteurs de «simples » autour de Milly qui ont conservé une structure artisanale et/ ou bio, s'ajoute le groupe familial Darbonne-Darégal implanté à Milly depuis 1887, devenu leader 
mondial des herbes aromatiques culinaires déshydratées et surgelées, qui passe contrat sur des centaines d'hectares avec des céréaliers ou des maraîchers de la région.

11. Une enquête de fréquentation sera menée dans le cadre d'Agrige pour aller plus loin dans l'analyse, notamment en fonction des périodes (semaine/week-end, vacances).

12. Ce résultat est le fruit d'enquêtes (entretiens semi-directifs et relevés de terrain) réalisés sur plusieurs territoires (plaine de Versailles, territoire de la Côte ouest de l'île de la Réunion...) dans le cadre du programme de recherche PFP-RIO cité précédemment.

13. Une cinquantaine d'entretiens semi-directifs menés auprès des habitants et des élus dans ce TAU.

14. D'autres enquêtes sont prévues dans le cadre du PSDR pour évaluer les formes d'habiter dans les territoires agriurbains et les appropriations paysagères selon un échantillonnage plus fin des populations enquêtées afin de cerner au plus près les représentations des habitants et la modification de leurs pratiques dans ces territoires.

15. Cette thèse a fait l'objet d'une publication dix ans après (Yasmmeen, 2006).

16. L'élevage n'apparaît pas ou fort peu dans les enquêtes sans doute du fait de sa quasidisparition. Il serait intéressant de comparer avec d'autres régions : qu'en est-il des prairies et de l'élevage dans l'idée habitante de paysage alimentaire?

17. Ces représentations ont été repérées dans des enquêtes menées dans les TAU (Faramond et al., 2015 ; Gautron, 2017).

18. Cf. projet du Plan vert régional d'île-de-France (1995), repris dans le SDRIF de 2013.

19. Le phénomène est difficilement mesurable au vu des statistiques officielles : le recensement général agricole (RGA) date de 2010 et nombre de ces microfermes sont en deçà de la surface minimale d'installation reconnue. Seules les installations faites par la Région dans les périmètres régionaux d'intervention foncière sont documentées (appels d'offre de la Safer). Le programme Agrige entend étudier plus avant ce phénomène (Gautron, 2017).

20. On est là face à un autre métier que celui d'agriculteur au sens de producteur de denrées alimentaires.

21. Nous faisons l'hypothèse que l'accueil du public fait l'objet de mises en scène moins élaborées dans les territoires ruraux « ordinaires » où l'emprise des grandes métropoles est moindre. Les dispositifs y sont souvent simples et parfois même sommaires, organisés avec les moyens du bord, et en continuité avec l'entité de la ferme ; en outre, le vendeur est la plupart du temps le producteur. Toute chose qui permet au client/cueilleur de nouer une relation de plus grande proximité avec l'agriculteur.

22. En 2012, le nombre de pages consacrées aux espaces agricoles et à l'agriculture a été multiplié par 4 ou 5 par rapport à 1994. Les pages majeures dans le SDRIF 2012 sont dans les parties 2 (p. 42, $66,74)$ et 4 (p. 126-127 et 146 et sq).

23. Pourtant, dans les débats scientifiques, selon une définition avant tout fonctionnelle, l'agriculture urbaine intègre les agricultures intra-urbaine et périurbaine dès lors qu'elles sont à destination des urbains (Aubry, 2014).

24. Une résistance à lire à l'aune de la rupture de l'après-guerre et de l'ère du productivisme quand l'agriculture devient un métier en place et lieu d'un état (Mendras, 1992). 


\section{RÉSUMÉS}

Les territoires agriurbains (TAU), derniers nés des territoires de projet en région Île-de-France (1999), consacrent la prise en compte des espaces agricoles et de leurs paysages dans la construction de l'urbain. Cet article interroge les figures territoriales et paysagères portées par les acteurs partie prenante, le paysage étant entendu dans ses deux dimensions matérielle et immatérielle. Construits au départ autour d'un projet d'agriurbanisme - qui a légitimé leur création - les TAU ont promu deux formes paysagères emblématiques que sont la lisière urbaine et le sentier d'interprétation agricole. La montée des préoccupations alimentaires dans les TAU modifie quelque peu ce projet. Les auteures questionnent les nouveaux motifs, notamment nourriciers, en voie d'apparition et leur éventuelle contribution à faire advenir au rang de paysage des territoires de franges ordinaires, longtemps (et encore aujourd'hui) perçus comme "sans qualités ». S'inscrivant à la croisée de la géographie et de la sociologie, le parti pris est d'essayer de mettre au jour certains des présupposés au fondement des actions et des discours à l'œuvre sur ces territoires et présentés comme allant de soi.

Agri-urban areas, the latest-born "project territories" (in French, territoires de projet) in the Îlede-France region (1999), integrate farming spaces and landscapes in urban development. This article looks at the territorial and landscape features promoted by the stakeholders, the term landscape being understood in both its tangible and intangible dimensions. Agri-urban areas, which were initially formed around agri-urban development projects which legitimated their creation, also widely promoted two emblematic forms of landscape that are the urban fringe (in French, lisière urbaine) and the agricultural trail (in French, sentier d'interprétation agricole). Increased focus on food production in agri-urban areas has somewhat changed these initial projects. The authors examine the new motives, namely that of food production, which are emerging and their possible contribution towards raising to the rank of landscape ordinary urban fringes which have been for a long time, and are still today, perceived as devoid of any notable "qualities". The approach used here, which is a cross between a geographical and a sociological study, attempts to bring to light certain presuppositions concerning the principle behind the actions and initiatives in the development of these areas which are presented as selfevident.

\section{INDEX}

Keywords : agri-urban areas, agricultural landscape, food-producing landscape, legitimation, social ties, landscape features.

Mots-clés : territoires agriurbains, paysage agricole, paysage alimentaire, discours de légitimation, lien social, figure paysagère

\section{AUTEURS}

\section{MONIQUE TOUBLANC}

Monique Toublanc est sociologue, ingénieur paysagiste, maître de conférences à l'École nationale supérieure de paysage de Versailles-Marseille, Laboratoire de recherche en projet de paysage (Larep).

m.toublanc[at]ecole-paysage[dot]fr 


\section{MONIQUE POULOT}

Monique Poulot est géographe, professeur à l'université de Paris Nanterre, Mosaïques-UMR Lavue.

monique.poulotmoreau[at]parisnanterre[dot]fr 